\title{
The value of accurate clinical assessment in the surgical management of the lumbar disc protrusion
}

\author{
R S C KERR, T A CADOUX-HUDSON, C B T ADAMS \\ From the Department of Neurological Surgery, The Radcliffe Infirmary, Oxford, UK
}

SUMMARY One hundred patients with lumbar disc protrusions were studied. Thirty six "control" patients were admitted in the same time period with low back pain and sciatica but with subsequently "normal" myelograms and no surgery. The aim of this paper was to relate history and clinical signs to the myelograms and surgical findings. Ninety nine per cent of our patients presented with sciatica (controls $94 \%$ ). The most frequently found sign in patients with a disc protrusion was reduction of ipsilateral straight leg raising $(98 \%)$. However, $55 \%$ of controls also showed this sign. There were three signs that, when present, particularly indicated a disc protrusion; "crossed straight leg raising" (pain on contralateral straight leg raising), measured calf wasting and impaired ankle reflex: the latter being especially indicative of an L5-S1 disc protrusion. There were two further important signs, weakness of dorsiflexion of the foot and scoliosis of the lumbar spine.

However, such signs occurred in about half the patients and so clinical diagnosis in the remaining half depended on obtaining a good history of sciatica, and paying due regard to severity of the pain, the mobility of the patient, the ability and desire to work and the overall personality. Satisfactory results of surgery simply depend on finding and removing a definite disc protrusion. Using these methods of selection, $98 \%$ have returned to their original employment, $86 \%$ within 3 months of the operation. For a patient with no abnormal signs and a normal myelogram, surgical treatment should not be advised.

The operation of lumbar laminectomy and the removal of a protruded intervertebral disc is now an extremely common procedure, some 200,000 being performed in the USA each year. ${ }^{1}$ The success rate, assessed by the number of patients achieving loss of pain and being able to return to work, varies from $60 \%$ to $99 \%$. $^{1-4}$

For a patient suffering low back pain and sciatica, deciding which mode of therapy to employ depends very much upon the underlying pathology. If due to a protruded intervertebral disc, resolution of the pain will occur in the majority of cases using conservative methods of treatment. ${ }^{145}$ The surgical removal of the protruded disc is indicated acutely in those who have symptoms and signs of cauda equina compression, and electively ${ }^{6}$ in those who fail to respond

Address for reprint requests: $\mathrm{Mr}$ C B T Adams, The Radcliffe Infirmary, Oxford OX2 6HE, UK.

Received 2 December 1986 and in revised form 24 June 1987. Accepted 26 June 1987 to conservative therapy and have evidence of a definite protrusion.

This study was undertaken in an attempt to delineate which criteria are the most reliable indicators of a lumbar disc protrusion.

\section{Method}

A retrospective study of 100 consecutive cases of protruded lumbar intervertebral discs, assessed and treated at The Radcliffe Infirmary, was carried out. In addition 36 patients suffering with back pain and sciatica, but relatively normal myelograms, were used for comparison. All the cases were reviewed preoperatively by one of the consultant staff. The operations were performed by one of a total of six surgeons, using a Wilson frame, and exposure of the disc by hemilaminectomy.

From each case, details of age, sex, duration of symptoms including the distribution of the pain and the presence of tingling and numbness, were recorded. The physical signs on examination were noted, the examination having been directed towards three specific areas, signs related to the back, tension signs and neurological signs in the legs. The maximum calf círcumference was measured and a difference 
of more than $1 \mathrm{~cm}$ was considered significant. The power of dorsiflexion of the foot was assessed by asking the patient to dorsiflex the foot against resistance starting in the plantar flexed position; this "kinetic" assessment being more accurate than the commonly used test with the foot "locked" in dorsiflexion.

All the patients had a metrizamide myelogram (except one who had a CT myelogram) and these radiographs were assessed in the light of the history and physical findings. In all cases, an examination of the conus was included. Particular note was taken of the position of the protruded disc and its relation to the position of the last mobile joint. The radiographs were also examined for the presence of a transitional vertebra.

From the operation notes, the anatomy of the protrusion was noted, each one being graded according to the degree of protrusion, Grade 1 a protrusion with annular continuity, Grade 2 a protrusion with annular rupture and Grade 3, a sequestered fragment. All grades involve distortion of the nerve root.

Patient follow up was achieved by out-patient assessment and telephone. A total of 74 out of the 100 patients were contacted by telephone. Results were assessed in terms of work record, and changes in sciatica and back pain expressed as a pain score ranging from 0 to $10(0=$ no pain and $10=$ pre-operative pain). Statistical analysis was performed using a $\chi^{2}$ test with a Yates correction.

\section{Results}

Included in the series of 100 cases were $50 \mathrm{~L} 4 / \mathrm{L} 5$ protrusions, $47 \mathrm{~L} 5 / \mathrm{S} 1$ protrusions, and $3 \mathrm{~L} 3 / \mathrm{L} 4$ protrusions (table 1). The average duration of symptoms prior to surgery was 21 weeks (control 28, 4-104), ranging from under 4 weeks to over 120 , and the average age at presentation was 40 years (control 41 ).

Ninety nine per cent (control $94 \%$ ) of the patients presented with sciatica and of those $10 \%$ (control $11 \%$ ) had bilateral pain. Of the $10 \%$, four had central protrusions and three sequestrated fragments. Sensory symptoms of tingling and numbness were found in $30 \%$ (control $42 \%$ ), 17 in the $\mathrm{S} 1$ dermatome and 13 in the L5 dermatome, these corresponding to the levels of root compression.

The findings on examination were recorded in relation to three specific areas, back signs, tension signs and neurological signs in the legs (fig 1). A scoliosis was found in $63 \%$ (control $11 \%$ ) patients, 29 L4/L5 and $34 \mathrm{~L} 5 / \mathrm{S} 1$ discs. Sciatic pain was elicited in $98 \%$ (control $55 \%$ ) on elevating the ipsilateral limb and $43 \%$ (control $4 \%$ ) on elevating the contralateral

Table 1 Sex distributions of disc protrusion

\begin{tabular}{lccc}
\hline & Male & Female & Total \\
\hline Prolapsed L3/L4 disc & 2 & 1 & 3 \\
Prolapsed L4/L5 disc & 31 & 19 & 50 \\
Prolapsed L5/SI disc & 22 & 25 & 47 \\
\hline
\end{tabular}

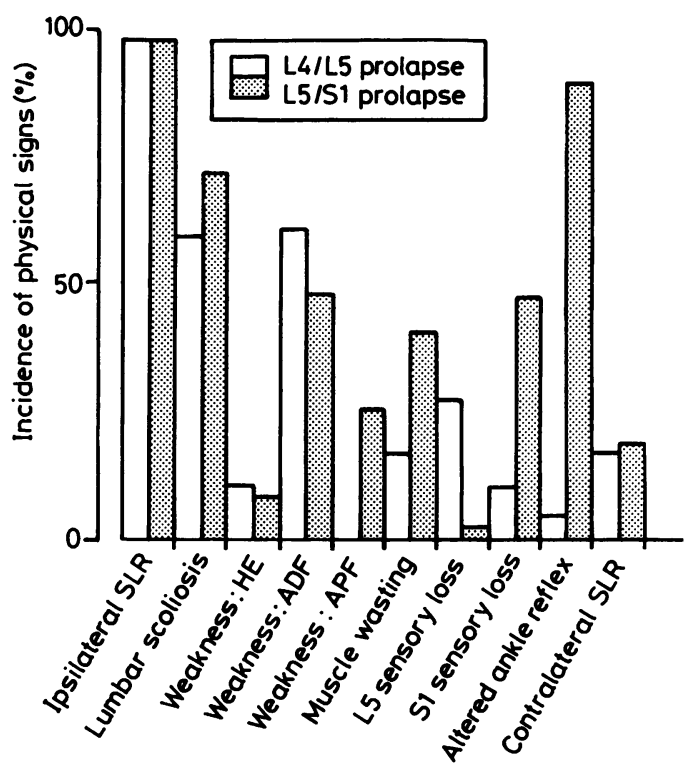

Fig 1 The incidence of the physical signs associated with disc protrusions at the L4/L5 and L5/S1 levels: APF, ankle plantar flexion; $A D F$, ankle dorsiflexion; $H E$, hip extension.

limb (21 L4/L5 and 22 L5/S1). As regards neurological signs (fig 1), all the cases were examined for alterations in power, muscle wasting, reflex changes and sensory changes. Significant measured calf wasting was found in $29 \%$ (control $4 \%$ ). A reduction in the power of hip extension was found in $10 \%$ cases (control 4\%), six due to L4/L5 protrusion and four due to $\mathrm{L} 5 / \mathrm{S} 1$. Reduced power in ankle dorsiflexion was demonstrated in $54 \%$ (control $11 \%$ ) cases, 30 due to $\mathrm{L} 4 / \mathrm{L} 5$ protrusion, 23 due to $\mathrm{L} 5 / \mathrm{S} 1$ and one due to L3/L4. Reduced power in ankle plantarflexion was demonstrated in $13 \%$ (control $0 \%$ ) cases, all of which were due to disc protrusion at the L5/S1 joint space. Alterations in the ankle reflex were noted in a total of $48 \%$ (control $11 \%$ ) cases, 41 of these patients suffering an $\mathrm{L} 5 / \mathrm{S} 1$ disc protrusion and seven an L4/L5 disc protrusion. These results indicate that changes of the ankle reflex are found in $87 \%$ of cases of $\mathrm{L} 5 / \mathrm{S} 1$ disc protrusion and only $12 \%$ of $\mathrm{L} 4 / \mathrm{L} 5 \mathrm{disc}$ protrusion, a highly significant result $(\mathrm{p}<0.01)$. Of the seven patients with a L4/L5 protrusion demonstrating ankle reflex changes, four were found to have central discs, one an element of lumbar canal stenosis and one was a case of re-exploration due to failure of the initial operation. A total of six (13\%) cases of disc protrusion at the L5/S1 level had a preserved ankle reflex. At operation, it was found that in two cases there was a sequestered fragment that had migrated distally down the spinal canal into the sacrum, in one was a central disc and in another a Grade 1 prolapse. Only two out of the 47 cases had a Grade 2 postero- 
Table 2 Comparison of clinical signs

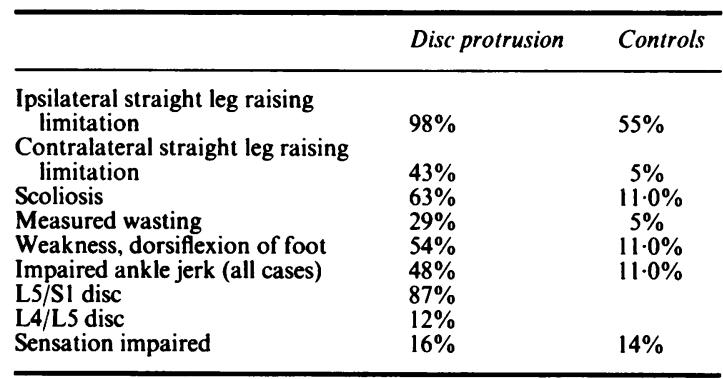

lateral prolapse at the L5/S1 level, with a preserved ankle reflex. Reduced sensation in the L5 dermatome was observed in $16 \%$ (control $14 \%$ ) patients, only one of whom had a L5/S1 disc protrusion and this was a grade 2 prolapse. Abnormalities in the $\mathrm{S} 1$ dermatome were noted in $28 \%$ (control $14 \%$ ) patients and of these, six were due to L4/L5 disc protrusions, one associated with a grade 2 protrusion, two with central discs, two with sacralised vertebrae and only one with a postero-lateral L4/L5 protrusion.

Results of treatment (table 3) showed that $98 \%$ of patients were able to return to work, $86 \%$ of these within 3 months. Sixty two percent of the patients enjoyed an immediate total loss of sciatic pain. Only $41 \%$ have totally lost their back pain (table 4 ).

The mean pain score for the 74 patients followed up since surgery (fig 2) shows a drop from 10 to $1 \cdot 3$ for sciatica, and 1.8 for the back pain. In the control group $22 \%$ were still suffering sciatica at follow up, and $64 \%$ back pain. Only one patient from the control group suffered a post myelogram headache, with readmission for recovery.

None of the patients reported that either the back pain or the sciatica had become more severe as a result of the surgery. Only two patients from the control group were readmitted for surgery at a later date, one with L3/L4 stenosis, the other with an L4/L5 disc protrusion.

Table 3 Time interval (weeks) following surgery before returning to work

\begin{tabular}{lcc}
\hline & Number & $\%$ of total \\
\hline Under 12 & 64 & 86 \\
$13-24$ & 4 & 5 \\
Over 24 & 6 & 7 \\
Unable to work & 2 & 2 \\
\hline
\end{tabular}

Table 4 Patients totally relieved of pain (\%)

\begin{tabular}{lll}
\hline & After operation & Now \\
\hline Back pain & 33 & 41 \\
Sciatica & 62 & 86 \\
\hline
\end{tabular}

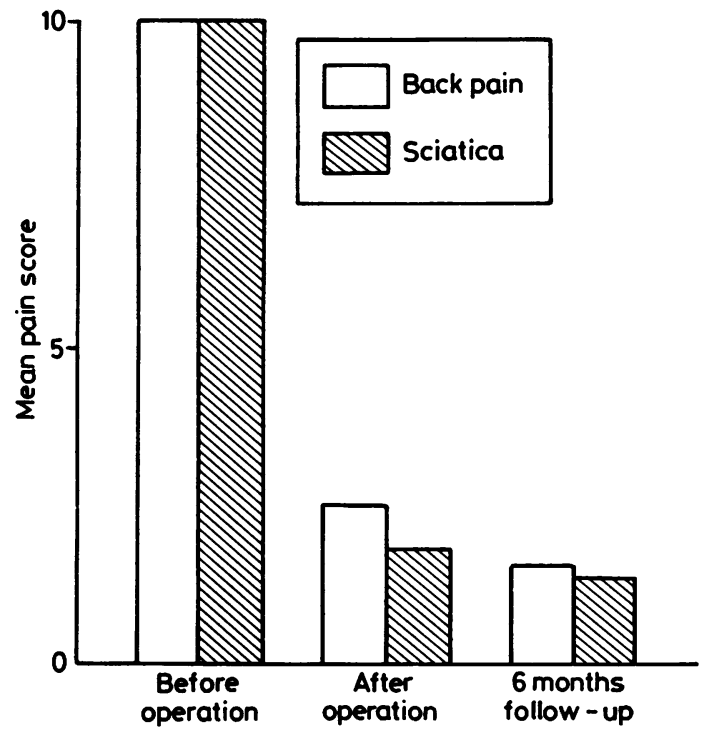

Fig 2 Graph showing alterations in the mean pain score following surgery.

\section{Discussion}

Successful surgery for lumbar disc protrusion depends upon identifying those patients with a significant protrusion, determining the level of the protrusion and removing the pathological disc. Since the initial descriptions of lumbar disc protrusion by Barr in $1937,,^{7}$ many articles have been published describing the importance of various clinical signs resultant from disc protrusion. In an attempt to overcome this problem, some have ascribed a grading system, in the form of a "Predictive Score Card", to selected features in the history and examination, and the myelogram. ${ }^{8}$ The higher the score achieved, the greater the chance of a satisfactory outcome.

In this study, the symptoms and signs of each case have been assessed with the prior knowledge that all the patients had a lumbar disc protrusion.

By definition, for a patient to have sciatica, they must describe pain in the distribution of the sciatic nerve. Ninety nine per cent of the cases reported here described such pain. In many, although associated with back pain, the leg pain was the predominant symptom. Sensory symptoms, in this series and in others, ${ }^{69}$ were found to be of limited value.

Lumbar disc protrusions seem to occur around $\mathbf{4 0}$ years of age, with a slight male predominance. Other reported series have suggested that the L4/L5 interspace is affected more commonly than the L5/S1.910 Although our results support this, the figures were not statistically significant.

The value of conservative therapy is obvious with 
many cases resolving without requiring surgery. Resolution usually takes place within 6 weeks. ${ }^{7}$ The average duration of symptoms before presentation in this series was 21 weeks, time spent away from work, resting at home. With better patient selection, this period of suffering could be reduced considerably, many people being able to return to work within a few weeks of surgery. ${ }^{7}$ This is supported by this study, with $86 \%$ of the patients returning to work within 12 weeks.

Distortion of a nerve root by an intervertebral disc protrusion will produce signs that may be generalised, or specific for that level. The most consistent general sign was that of limitation of straight leg raising, found in $98 \%$ (control $55 \%$ ) of patients. Much emphasis has been placed on this physical sign, ${ }^{1248-11}$ but its value in the diagnosis of a prolapsed disc is as a support for the history. To find a limitation of straight leg raising in a patient with no symptoms of sciatica is not an indication on its own to proceed to myelography. Many patients found not to have a disc prolapse show a limitation of straight leg raising (control 55\%). ${ }^{12}$ The presence of crossed pain with straight leg raising, although not found so frequently, was often associated with a grade 3 prolapse, and is of greater value. ${ }^{11}$

Of the specific signs, it has been suggested that alterations in the ankle reflex cannot always be attributed to a L5/S1 disc protrusion. ${ }^{12}$ From the results reported above, it is apparent that this is not the case. All the L5/S1 protrusions had a reduced or absent ankle reflex except for six. In those where the reflex was preserved, the protrusion had occurred in such a way as to avoid distortion of the $\mathrm{S} 1$ nerve roots, for example, a distal migration of the sequestered fragment or a central protrusion. Applying this principle to disease at the L4/L5 level, those cases where the ankle reflex was affected were associated with either a central protrusion, co-existent lumbar canal stenosis or re-exploration due to failed surgery, with evidence of $\mathrm{S} 1$ nerve root distortion. The ankle reflex is therefore of vital importance. Although a difficult reflex to elicit, unless the patient is relaxed and limb held in the correct position, its presence or absence will lend considerable support to achieving a correct diagnosis.

Although table 2 shows that only about half the disc patients showed physical signs, their importance is that, when present, such signs usually do indicate a disc protrusion. In contrast, the control group rarely showed signs, especially pain on contralateral straight leg raising and significant measured wasting. The latter is a particularly important sign for, like the ankle reflex, it is an objective sign independent of the cooperation of the patient. In this aspect of clinical neurosurgery perhaps more so than any other, can the doctor least expect the wholehearted cooperation of the patient. We therefore commend the use of the tape measure, and correct technique for estimating power of dorsiflexion, in assessing these patients. Locking the foot in dorsiflexion, as advocated by most neurologists, will miss the more moderate levels of weakness.

Myelography, using water soluble contrast media such as metrizamide, forms an integral part of the immediate pre-operative assessment for all patients, but should only be performed if surgical treatment is indicated. In the light of the findings so far described, correct interpretation of the radiographs can only be achieved with knowledge of the clinical signs, details of which must be conveyed to the radiologist. For a complete examination, views of the conus should always be taken so as not to miss some other lesion causing lower motor neuron signs, such as a spinal angioma or tumour at a higher level. Where myelography has failed to confirm the diagnosis, CT myelography may be of value, especially for demonstrating laterally placed discs. CT scanning without the addition of intra-thecal contrast is unreliable, with many false positive and false negative results, ${ }^{13}$ and should not be relied upon for advising surgery for treatment of lumbar disc protrusion.

A review of 100 patients who did not enjoy a successful outcome following lumbar disc surgery ${ }^{14}$ revealed that $78 \%$ did not suffer sciatica preoperatively with no signs suggesting nerve root compression. From our results one can conclude that the history is of paramount importance. Alteration of the ankle reflex due to $S 1$ nerve root compression from an L5/S1 prolapse is the most consistent physical finding. With L5 nerve root compression from an L4/L5 protrusion, there may be very little in the way of physical findings. But when wasting of the calf, or weakness of dorsiflexion or a scoliosis of the lumbar spine are found, such signs are particularly indicative of a disc protrusion. These signs only occur in approximately half the patients but when they are found they are of significance, since they are rarely found in the control group.

That $98 \%$ of patients have obtained an excellent result emphasises the fundamental truism that if a definite disc protrusion is found and removed, the patient is relieved of pain.

These results have three further corollaries. The first is that a merely bulging disc is not pathological and should not be removed. Secondly, that all these patients rendered pain free inevitably have adhesions around the nerve roots. Thus extrathecal adhesions are not a cause of pain, making the placing of fat "grafts" around the nerve roots to "avoid" extrathecal adhesions unnecessary. Such adhesions invariably occur and if the patient still has postoperative pain then the surgeon would do better to pay more 
regard to the correct selection of patients than deluding himself and his patient about adhesions. Finally, such results obviate the need for fusion following disc protrusion. Fusion is wholly unnecessary if a definite disc protrusion has been removed. Much more necessary is to emphasise the importance of back extension exercises. Every patient in our department is discharged with a leaflet describing exactly what exercises should be done. In our experience the importance of re-education of the back muscles following surgery (and thereby improving the stability of the back) has not been sufficiently emphasised by most surgeons and accounts for some of the "failed backs".

Mixter and Barr in 1941 wrote that "There is no easy road to correct diagnosis which can supplant the time honoured method of careful history taking, complete physical examination, and the evaluation of that information in the light of appropriate laboratory tests". ${ }^{8}$ In order to achieve higher success rates, perhaps we should heed these words more closely when selecting patients for this commonly performed procedure.

We thank Mr M Briggs and Mr PJ Teddy for allowing us to include their patients in this series.

\section{References}

1 Herron LD, Turner J. Patient selection for lumbar laminectomy and discectomy with a revised objective rating system. Clin Orthop 1985;199:145-52.
2 Spangfort EV. The lumbar disc herniation: A computeraided analysis of 2,504 operations. Acta Orthop Scand 1972;142suppl:1-95.

3 Jackson RK. The long term effects of wide laminectomy for disc excision. J Bone Joint Surg 1971;53B:609-16.

4 Dunkerly GS. The results of surgery for low back pain due to presumptive prolapsed intervertebral disc. Postgrad Med J 1971;47:120-8.

5 Van Loon L, Hoogmartens M. Results obtained with operative treatment of sciatica. Acta Orthop Belgica 1977;43:647-52.

6 Kosteljanetz M, Esperson JO, Halaburt H, Militic T. Predictive value of clinical and surgical findings in patients with lumbago-sciatica. A prospective study. Acta Neurochir 1984;73:67-76.

7 Barr JS. "Sciatica"caused by intervertebral-disc lesions. A report of forty cases of rupture of the intervertebral disc occurring in the low lumbar spine and causing pressure on the cauda equina. $J$ Bone Joint Surg 1937;19:323-42.

8 Finneson BE. A lumbar disc surgery predictive score card. Spine 1978;3:186-8.

9 Barr JS, Mixter WS. Posterior protrusion of the lumbar intervertebral disc. J Bone Joint Surg 1941;23A: 444-56.

10 Bartlett J. The problem of sciatica. Practitioner 1970;204:529-36.

11 Kortelainen P, Puranen J, Koivisto E, Lahde S. Symptoms and signs of sciatica and their relation to the localisation of the lumbar disc herniation. Spine 1985;10:88-92.

12 Sprangfort E. Lasegue's sign in patients with lumbar disc herniation. Acta Orthop Scand 1971;42:459-60.

13 Byrd SE, Cohn ML, Biggers SL, Huntington CT, Locke GE, Charlse MF. The radiographic evaluation of the symptomatic postoperative lumbar spine patient. Spine 1985;10:652-61.

14 Fager CA, Friedberg SR. Analysis of failures and poor results of lumbar spine surgery. Spine 1980;5:87-94. 